
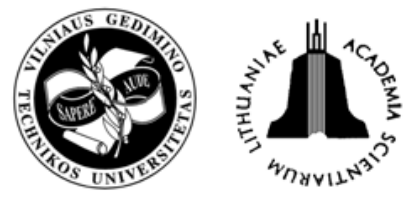

\title{
THE “GREEN WAVE” MODE PRODUCTION ON THE TWO-LANE HIGHWAYS DURING THE CONSTRUCTION WORKS TIME PERIOD
}

\author{
Alexander Berezhnoy ${ }^{1}$, Alexander Grakovsky ${ }^{2}$, Alexey Nesterov ${ }^{3}$ \\ Transport and Telecommunication Institute, Lomonosova 1, LV-1019 Riga, Latvia \\ E-mails: ${ }^{1} a v b @ t s i . l v ;{ }^{2} a v g @ t s i . l v ;{ }^{3}$ alenes@tsi.lv
}

Received 16 May 2007; accepted 10 September 2007

\begin{abstract}
In the paper, the problem of a bi-directional "green wave" mode production is considered for the vehicle flows motion on two-lane highways during the conducting of construction works. The solution of the given task allows to develop practical recommendations for the sphere of the construction works management on two-lane roads and country highways, and also to raise traffic control efficiency in the pointed conditions. The comparative analysis of possible traffic regulation modes for the set scheme of road construction works sites and the estimation of their efficiency is performed, the test of a hypothesis regarding the possibility of a bi-directional "green wave" mode realization is carried out, and calculation of traffic lights signal control phases time is performed.
\end{abstract}

Keywords: intelligent transport systems, traffic control, the "green wave" mode, traffic control effectiveness evaluation.

\section{Introduction}

One of the typical situations that drivers are forced to face in a daily life is regarded to be roads construction. Especial inconvenience in case of road construction is traffic flow motion regulation on two-lane sites of road. A general negative consequence of this situation is the congestion of extensive queues leading to formation of traffic jams on two-lane highways during the time periods of construction works.

The number of possibilities for traffic control on a two-lane highway in terms of construction works is not so big. One of them is a widespread full blocking of traffic, placing road information signs concerning a possible detour to avoid construction works zone.

Other known way consists in partial blocking of road and installation of traffic lights before the entrance to a repair site of the road (Fig. 1). Extent of a construction works site can differ. There also exists quite a frequent practice to carry out construction works on the several sites of road located one after another at once [1].

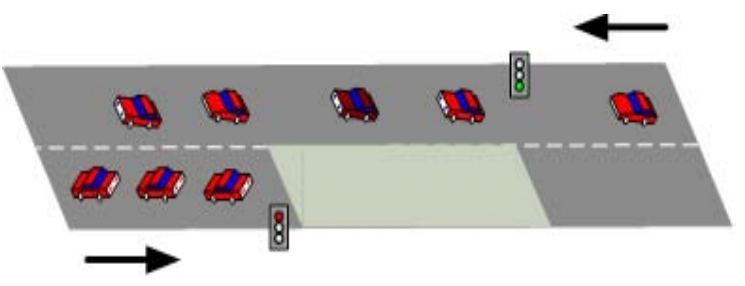

Fig. 1. Installation of traffic lights for arranging a sequenced traffic flow travel mode
The goals for conducting of construction works put forward indirect requirements to topology of a repair site. In particular, during the construction works time period, simultaneous expansion of road lanes width is practiced, though anyway, the width of a lane $w$ in each direction is set much less than extents of construction works sites and intervals between the next repair zones $l>w, c>w$. The neighboring construction works zones should be distanced farther enough from each other in order to ensure unobstructed travel of counter streams at the moment of their departure from a site of one-strip traffic (Fig. 2).

The basic problems arising from the organization of traffic are the uncertainty of a choice of the control facilities, allowing for changing a situation, and an existing intuitive estimation for a choice of traffic lights signal control time phases.

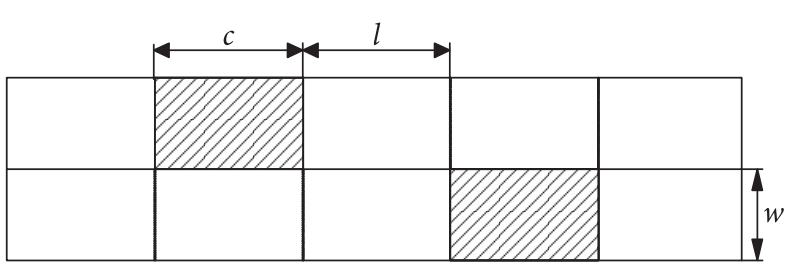

Fig. 2. Topology of repair sites during carrying out of construction works on a two-lane highway 


\section{The analysis of traffic regulation modes at the time period of construction works on two-way highways}

\subsection{Sequential gating of traffic streams in each of directions}

The natural decision on the traffic organization in conditions of construction works is the traffic lights signal control that allows providing sequential traveling of vehicles on an accessible lane. In case of traffic lights installation there is a problem of a time phase choice, given for the motion in each of directions. Usually, for a country highway the fixed waiting time of the car before a traffic light is often established - for example, it is often set to 5 minutes. But a choice of the given time interval of the traffic light red signal is unreasonable from the point of reaching the peak efficiency for traffic regulation.

Planning of the organization of road construction works will include a choice of two categories of parameters:

1. Topological (the general extent of a constructed road site, quantity and extent of separate repair zones, distances between the next construction works zones, a correlation of distances between zones and extents of construction works zones).

2. Traffic lights signal control (the general length of a signal control cycle, time of a traffic light green and red signals) [1].

Topological conditionality is shown in time necessary for travel through the entire construction works corridor. Red light signal time duration should take into account an additional stock for leaving of one-lane road site by the last vehicle that moves at a speed that is allowed on construction works road sites till the moment of change of a motion direction on the given lane. The mentioned time stock leads to an even greater waiting time from drivers of a counter traffic stream.

Cars pass a construction works site in a chainpacked mode. By increasing of the traffic light green signal time at the entrance to a construction works zone, the number of passing vehicles for one cycle of traffic lights signal control also increases. Thus, the pack of cars has time to reach a high-speed mode close to a maximum admissible, and the share of time falling to the process of acceleration and a set of speed is reduced as compared to the motion time in the cruise travel mode. On the other hand, the vehicles queue waiting time is increased for traveling in the opposite direction.

The case when the whole chain of construction works zones is blocked, and the motion has the unidirectional character during the traffic light green signal cycle, can be interpreted as production of the unidirectional "green wave" mode (Fig. 3).

Major problems of the construction works zone sequential travel on a two-lane highway are rooted in the non-uniform accumulation of queues in each of directions, because of the existing difference in the real-life vehicles flow intensity for the different sides. The initial queues extent as well as the average flow motion velocity make a strong influence on the drivers' psycho emo-

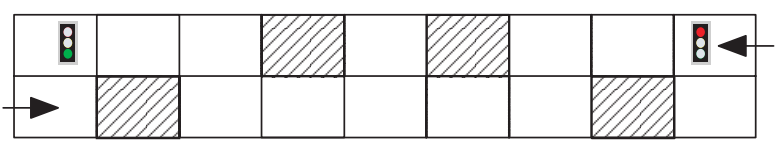

Fig. 3. Traffic regulation for the travel through a chain of road construction works sites in a mode of the unidirectional "green wave"

tional condition connected with long-term waiting and reflecting on their behavior [2].

\subsection{Opportunities of realization of a bi-directional "green wave"}

It is possible to assume, that there may also exist the other form of passing through the construction works corridor where repair zones are settled down in a way of providing for vehicles traffic streams an opportunity of simultaneous traveling in counter directions. We shall name such way of traffic regulation as a mode of a bi-directional "green wave".

We start with the assumption that it is necessary to set the construction works zones in a chessboard order. To make it more simple, at the beginning it is assumed that all construction works zones are identical in their length $c_{1}=c_{2}=\ldots=c_{i}=c$, distances between neighboring zones are equal to $l_{1}=l_{2}=\ldots=l_{i}=l$, and coincide with extent of repair zones $c=l$, i.e. we provide the observance of topological symmetry condition of projected construction works sites. In real life, it is usually chosen as the relation between the distance and zones extent, i.e. $l \geq c$.

Vehicle traffic flows control is realized by means of signal control. Traffic lights signal objects of two types are present - forming and confirming. The primary goal of operating traffic lights is the formation of car packs of necessary length. The first type traffic lights are only two and they are allocated to the entrance and final points of a construction works zones chain. Accumulation of a stream occurs at the first forming traffic light. Any additional forming traffic lights that are set beforehand at the entrances to a construction works zones chain are not required. The confirming traffic lights are present at the entrances to each of separately taken construction works zone. They operate in accordance with a set schedule repeating signal control cycles of the forming traffic light with the set time shift and provide the function of traffic safety, in case if any separately taken vehicle (or a group of vehicles) has failed to leave the site of a one-way traveling in due time .

On the basis of the traffic schemes analysis it is possible to formulate the following heuristic rules. The minimum of two consequent road construction works sites is required for a bi-directional "green wave" mode production. For the allocation of plenty of construction works zones $n$, it is desirable that the condition of frequency rate of their quantity to two was satisfied. In order to perform the synchronization of bi-directional traffic it is required to provide in a counter traffic streams simultaneous travel point for an interval between the following next parting packs of cars in three stages (two free and construction work zone, or two construction works and one free sites). It corresponds to the moment 
of the largest territorial distancing of the next vehicles pack from each other (Fig. 4). Introduction of any additional vehicle pack on an intermediate road site will lead to infringement of synchronization and impossibility of bi-directional synchronous traveling.

\subsection{Bi-directional "green wave" analytical model}

From the received description of the offered mode of bi-directional traffic it follows that the buffer zone in each of the directions, being at traffic lights, directly ahead of the beginning of the closed construction works zone will be filled with traffic on each fourth step of signal control. At the first step the pack of vehicles leaves the taken position on green light then, having passed a pack of cars, the traffic lights should be switched to a red signal for the given direction of traffic and remain in such mode before departure of the next vehicles pack for the subsequent three steps (Fig. 5).

For the safety reasons there is a restriction set to a maximum possible admissible speed [3]. The pack of vehicles moves at the speed not exceeding the maximum speed admissible in the space of entire construction works corridor. Thus, time spent for travel on one site of a line, will be estimated as:

$$
\Delta t=\frac{l}{v_{\text {constr }}},
$$

where $\Delta t$ - time of travel on a separate site, $v_{\text {constr }}-$ recommended or maximum possible admissible speed on a road site in terms of construction works, $l$ - extent of the site of a separate zone.

Then, under condition of equality of time for traffic lights green and red signals, time of a full signal control cycle will be $4 \cdot \Delta t$ (Fig. 5).

From the mentioned it follows that if the forming traffic light during three steps of a red signal accumulates a pack of vehicles, which length is limited to distance $l$, it is possible to write down, that:

$$
3 \Delta t \cdot \lambda \leq \frac{l}{x_{v e h}}
$$

where $\lambda$ - vehicles stream entrance intensity, $x_{v e h}-$ an average length of the car taking into account the distance to the following vehicle in a queue.

Differently, the quantity of cars in queue at the forming traffic light, accumulated during the red light cycle should not exceed the quantity of vehicles, which could be placed within in motion on a site between construction works zones for free travel of counter streams. In the case, if the queue appears longer than the stipulated condition, its surplus has no time to pass at a time of the traffic lights green signal.

Then,

$$
\lambda \leq \frac{l \cdot v_{\text {constr }}}{x_{v e h} \cdot 3 l}=\frac{v_{\text {constr }}}{3 x_{v e h}} .
$$

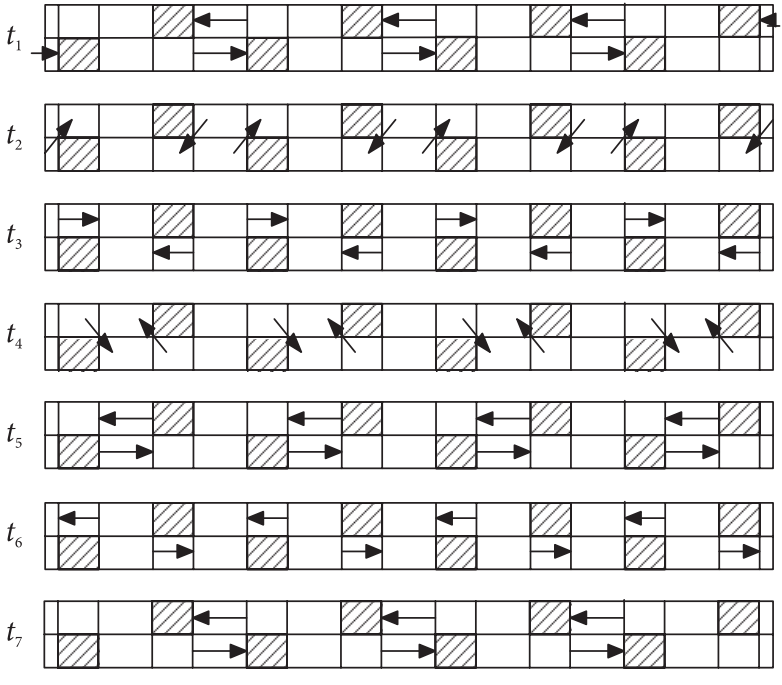

Fig. 4. Time diagrams of vehicle packs traveling in a bidirectional "green wave" mode on a two-lane highway during construction works time period

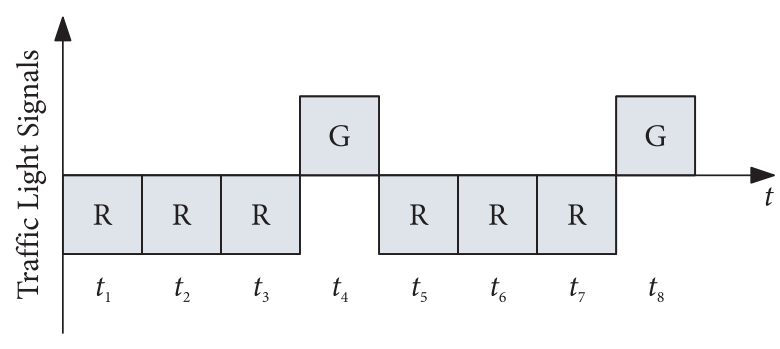

Fig. 5. The time diagram of forming traffic lights operation

$\mathrm{Or}$

$\lambda \leq \frac{v_{\text {constr }}}{3 x_{\text {veh }}}$.

Therefore,

$v_{\text {constr }} \geq 3 x_{\text {veh }} \cdot \lambda$.

The further detailed elaboration of model demands specification of requirements to the time interval allocated for providing of the guaranteed leaving of potential zones of collisions between vehicles and corresponding correction of traffic lights signal control time phases.

\subsection{Calculation of traffic lights signal control time phases}

For further practical adjustment of model, it is required to define the full time of a control cycle $T$, and separate traffic lights signal control time phases. In general form, $T$ develops from green, red and yellow signal times of the traffic light cycle:

$$
T=T_{G}+T_{R}+T_{Y}
$$

Thus, in order to create a safe travel time interval the condition $T_{G}<T_{R}$ should be carried out, since during the traffic lights red signal operation, all entered vehicles should have enough time to leave sites of a one-way traffic. Time of the traffic lights yellow signal $T_{Y}$ compiles 
$5-10 \%$ of the green light time and is also taking part in creation of a safe travel time period. Sometimes, for example, in a mode of a bi-directional "green wave" the traffic lights yellow signal can be completely excluded from the process of signal control.

Adjustment of the plan of signal control should be realized in a way of minimizing the total waiting time for vehicles drivers moving in both directions. In symmetric control mode such requirement can be provided only under condition of presence of continuous vehicle streams of identical intensity from both sides. In case of load changing from one of directions, an asymmetric control mode could be demanded. For a mode of a bi-directional "green wave", the time of the traffic lights signal control full cycle consists of 4 steps that correspond to time needed for traveling of 4 consequent road sites by one pack of vehicles. In this case, the length of a pack will be determined by such topological characteristic as the extent of a site between construction works zones. For simplification of the problem, at the beginning of a model construction it was accepted that all zones have identical length.

In order to define the travel time values required for passing a construction works zone by one car, we shall get benefit of the vehicles traffic flow motion average speed parameter usage

$$
\Delta t=\frac{L}{\bar{v}}=\frac{4 \cdot l}{\bar{v}} .
$$

However, in calculation it is necessary to consider time of travel of all pack, and not just the first vehicle. Time of travel in 4 zones by a pack of vehicles can be estimated by the last vehicle from a pack. At equal zones and the maximal length of the pack, the first vehicle will leave for the following zone at the moment of entrance of the last car to a pack in the current zone, i.e.,

$$
\Delta t=\frac{4 \cdot l}{\bar{v}}+\frac{l}{\bar{v}}=\frac{5 \cdot l}{\bar{v}}
$$

For the solution to the problem of the effective signal control of sequential traffic of transport on one lane it is necessary to take into account the intensity of traffic in each of directions, which in its turn depends on a lot of factors - demand for traveling in the pointed direction, possibilities of detours on the nearby roads, existing high-speed restrictions and so forth. The usual character of change in traffic traveling demand has a cyclic origin. The factor of seasonal prevalence, day of week and time of day define cyclic dependences.

For the specified case where average speeds of vehicle streams motion coincide, and also, the duration of the traffic lights green signal is identical in both directions, it is possible to construct the time diagrams showing periodicity of operating signals and time of reverse motion mode use at the selected site of the road (Fig. 6).

On the basis of observations or the measuring information it can become known that intensity of traffic on the planned construction works zone has a strongly asymmetric character. And, the value of factor of asymmetry is considerably higher for movement in one of directions in the morning, and in the evening the situation varies to the opposite. In this case it is more expedient to use soft-operated traffic lights, which realize several operation control plans and will allow operating transport streams in a more flexible way.

\section{Efficiency evaluation for the various modes of traffic regulation}

Generally, as efficiency we understand the degree of suitability of the certain means and methods of their use for the decision of solved tasks.

For the answer to a question on expediency of realization of this or that mode of traffic regulation on a constructed two-lane highway, it is necessary to allocate criteria of efficiency evaluation. As those it is possible to consider the following parameters:

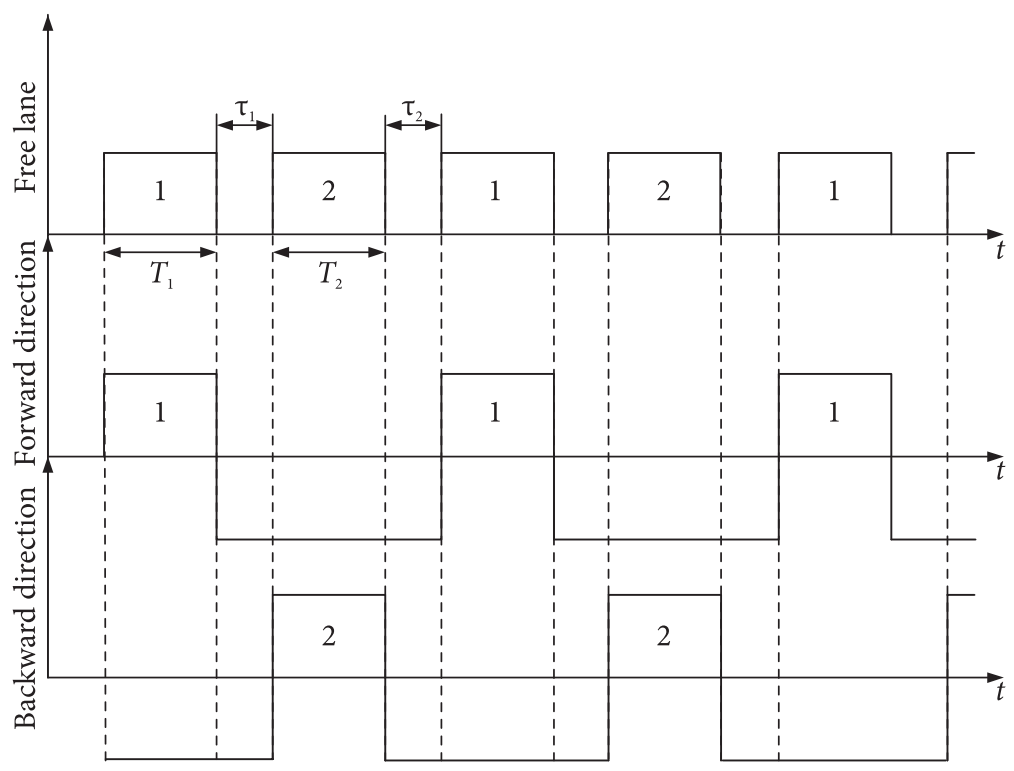

Fig. 6. The general case of time diagrams for the traffic regulation at the construction works zone of a two-way highway 
- total travel time in the construction works corridor;

- total waiting time for the beginning of vehicle packs motion in each of directions;

- average traffic speed on a line;

- loading index of construction works corridor;

- quantity of vehicles passed in both directions [4].

The most informative for an initial estimation of efficiency are the parameters of loading of the construction works corridor and the total waiting time for the beginning of vehicle packs motion in each of directions, because they are capable to give answers to the key questions on how much densely the road space will be used and how long it is necessary for the drivers of vehicles to wait for their turn to travel in each direction.

For a mode of sequential gating of vehicles traffic streams from different directions, it is possible to investigate two cases - sequential gating by one pack and sequential filling of all repair corridor in each of directions (Fig. 7).

For passing a construction works corridor (consisting of $k$ stages) by a separate vehicle pack, $k+1$ steps are necessary, where $k$ - is quantity of road sites, which are required to be passed. For example, in order to travel through a construction works corridor containing 4 repair zones (7 steps) by one vehicles pack in one direction, 8 steps of signal control are required. For consecutive filling and clearing by vehicles packs of the entire construction works corridor in one direction, $2 k$ steps will be required, and for travel of $2 k$ vehicles packs in both directions, $4 k$ steps will be required.

On traveling through similar construction works corridor in a mode of a bi-directional "green wave" (Fig. 8), the quantity of individual $k$ steps should correspond:

$$
k=3 r+(r-1)=4 r-1,
$$

where 3 - quantity of individual sites necessary for the organization of one pair of construction works zones, $r-$ quantity of construction works zones pairs, $r-1$ - quantity of steps between pairs of construction works zones. Thus, $2 r$ packs of vehicles will manage to travel per $k+1$ steps, in both directions.

Adjusting the model, we must note that the condition of frequency rate of the constructed zones quantity to two in a bi-directional "green wave" mode may not be observed. At a choice of odd quantity of the repair zones, one of confirming traffic lights will carry out the role of a forming traffic light.

Proceeding the definition of construction works corridor loading index, it is necessary to estimate at first the throughput of a constructed two-lane highway (quantity of vehicles packs divided by the number of the steps necessary for travel of the whole vehicles pack). For sequential travel, per 1 step, on the average, 0,5 vehicles packs succeed to pass. Similarly, in a mode of a bi-directional "green wave", for 1 step we obtain value of 0,5 vehicles packs that testifies that irrespective of the choice of a traffic control mode, the identical throughput of a construction works corridor is gained.

The construction works corridor loading index represents density of vehicles packs allocation in the construction works corridor of the given extent:

$$
K_{\text {surf }}=\frac{p}{k} \text {. }
$$

The time period of separate idle time in a buffer zone at the forming traffic light queue in case of sequential traffic motion will be defined by the extent of construction works corridor, i.e. the maximum quantity of waiting steps in one direction will be equal or a number divisible by travel time of the doubled number of construction works zones. While for a bi-directional "green wave" mode, maximal time of separate idle time of waiting before the forming traffic light will be equal or a number divisible by 3 steps of signal control.

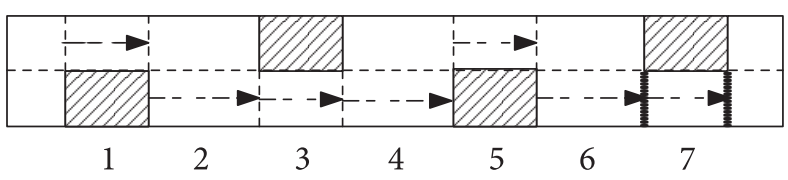

Fig. 7. An example of filling of construction works corridor for the travel in a sequential mode

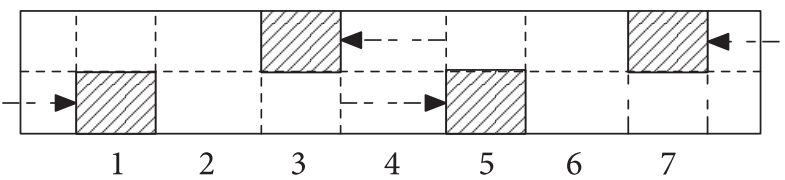

Fig. 8. An example of passing the construction works corridor in a bi-directional "green wave" mode

\begin{tabular}{|c|c|c|c|c|c|}
\hline $\begin{array}{l}\text { Quantity of } \\
\text { construction } \\
\text { works zones }\end{array}$ & $\begin{array}{l}\text { Quantity of } \\
\text { steps } k\end{array}$ & $\begin{array}{l}\text { Quantity of steps } \\
t \text { for passing in } \\
\text { unidirectional mode }\end{array}$ & $\begin{array}{l}\text { Number of vehicles packs } p \text {, } \\
\text { passing through corridor in } \\
\text { the unidirectional mode }\end{array}$ & $\begin{array}{l}\text { Number of steps } t \\
\text { for passing in a bi- } \\
\text { directional mode }\end{array}$ & $\begin{array}{l}\text { Number of vehicles } \\
\text { packs } p \text {, passing in a } \\
\text { bi-directional mode }\end{array}$ \\
\hline pairs $r$ & $k=4 \cdot r-1$ & $t=2 \cdot k$ & $p=k$ & $t=k+1$ & $p=2 \cdot r$ \\
\hline 1 & 3 & 6 & 3 & 4 & 2 \\
\hline 2 & 7 & 14 & 7 & 8 & 4 \\
\hline 3 & 11 & 22 & 11 & 12 & 6 \\
\hline 4 & 15 & 30 & 15 & 16 & 8 \\
\hline 5 & 19 & 38 & 19 & 20 & 10 \\
\hline 6 & 23 & 46 & 23 & 24 & 12 \\
\hline 7 & 27 & 54 & 27 & 28 & 14 \\
\hline 8 & 31 & 62 & 31 & 32 & 16 \\
\hline
\end{tabular}

Estimation of the general characteristics for passing the construction works corridor 
Resulting example of effectiveness evaluation for passing the construction works corridor in uni- and bidirectional "green wave" modes is shown in Table 1.

\section{Conclusions}

The hypothesis regarding the possibility of vehicles traffic flow regulation mode as a bi-directional "green wave" on a two-lane highway in the time period of construction works has the right to existence that proves to be true by the analytical calculations.

The comparative analysis of the traffic regulation modes efficiency on a constructed two-lane highway has shown that at equal throughput, the mode of bi-directional "green wave" essentially reduces waiting time for the vehicles packs in each of directions that promotes removal of a psycho emotional problem of drivers caused by long waiting in queues. The result of decrease in waiting time is especially significant for the case of construction works corridors of the large extent.

Practical recommendations on the choice of the traffic regulation mode and the order of carrying out of road construction works consist in the following: the chess order of placement of repair zones should be observed; the choice of the length of construction works zones conterminous with extent of sites of bilateral traffic between repair zones providing the traffic scheme symmetry should be realized. Certainly, ensuring the traffic safety also demands placement of additional follow-up intelligent transport systems providing immediate feedback inside the construction works corridor.

\section{References}

1. DREW, D. R. Traffic flow theory and control. McGraw-Hill Education, 1968. $384 \mathrm{p}$.

2. LIEU, H. Traffic flow theory. A State-of-the-Art Report. Revised Monograph on Traffic Flow Theory. FHWA, 1992. Available from Internet: <http://www.tfhrc.gov/its/tft/tft. htm>, 07.06.04.

3. SILYANOV, V. V. Traffic flow theory for road construction and traffic control (Сильянов В. В. Теория транспортных потоков в проектировании дорог и организации движения). Moscow: Transport, 1977. 303 p. (in Russian).

4. KOCHERGA, V. G.; ZYRYANOV, V. V. Evaluation and forecast of traffic flow parameters in intelligent transport systems (Кочерга В. Г., Зырянов В. В. Оченка и прогнозирование параметров дорожного движения в интеллектуальных транспортных системах). Rostov-on-Don: Rostov State Building University, 2001. 130 p. (in Russian). 